\title{
COMPARACIÓN ENTRE LOS PREDICTORES DEL ESTRÉS LABORAL SEGÚN EL NIVEL DE ACTIVIDAD FÍSICA, EDAD, GÉNERO Y ANTIGÜEDAD LABORAL EN UN GRUPO DE FUNCIONARIOS ADMINISTRATIVOS DEL SECTOR PÚBLICO COSTARRICENSE
}

\section{COMPARISON BETWEEN JOB STRESS PREDICTORS BASED ON PHYSICAL ACTIVITY, AGE, GENDER AND SENIORITY IN A GROUP OF ADMINISTRATIVE OFFICERS FROM THE COSTA RICAN PUBLIC SECTOR}

\author{
Christian Alberto Azofeifa Mora \\ Luis Solano Mora \\ Jorge Salas Cabrera \\ Héctor Fonseca Schmidt \\ Escuela de Ciencias del Movimiento Humano y Calidad de Vida-Universidad Nacional, \\ Costa Rica \\ azofeifa.cristian@gmail.com
}

\section{Resumen}

El objetivo de este artículo es relacionar y comparar los predictores del estrés laboral con el nivel de actividad física, la edad, la antigüedad laboral y el género en los empleados administrativos del sector público costarricense. Para ello, se encuestaron 395 sujetos pertenecientes a este sector. A los sujetos en estudio se les aplicó un cuestionario sociodemográfico (edad, género, tiempo laboral y estado civil) y para la evaluación de los predictores del estrés laboral se utilizó el cuestionario Job Stress Survey (JSS), compuesto por dos dimensiones (intensidad y frecuencia) cuyos predictores corresponden a la presión en el trabajo (PT), falta de apoyo social (FA), falta de organización (FO) y falta de realización personal (FRP). Para la determinación del nivel de actividad física, se utilizó el cuestionario internacional IPAQ, versión corta. Los resultados indicaron que los funcionarios masculinos presentan un nivel levemente superior en el nivel de actividad física en comparación con las mujeres; además, las mujeres muestran niveles superiores de presión en el trabajo como predictor de estrés. Asimismo, en aquellas personas cuyo nivel de actividad física es mayor, se muestran de forma subjetiva menores factores generadores de estrés, en comparación con los niveles de actividad física inferior. Se concluye que los altos niveles de inactividad física presentados pueden asociarse a mayores percepciones de estrés en el trabajo y la sobrecarga de roles puede ser un factor importante a considerar como fuente de estrés en el género femenino.

Palabras clave: estrés laboral, actividad física, funcionarios, género, edad, antigüedad.

\begin{abstract}
The purpose of this article is to relate and compare job stress predictors with physical activity levels, age, seniority, and gender in administrative employees in the Costa Rican public sector. A total of 395 individuals from this sector were surveyed. Subjects answered a socio-demographic questionnaire (age, gender, seniority, and marital status). In order to assess job stress predictors, the Job Stress Survey (JSS) was applied,
\end{abstract}


which was comprised of two dimensions (intensity and frequency) and including predictors such as pressure at work (PT), lack of social support (FA), lack of organization (FO), and lack of personal accomplishment (FRP). To determine the level of physical activity, a short version of the IPAQ was applied. Results indicate that male subjects have a slightly higher level of physical activity compared to female subjects. In addition, females show higher levels of job pressure as a stress predictor. Subjectively, the higher the physical activity level the lower the stressors, compared to lower levels of physical activity. We conclude that the high levels of physical inactivity exhibited may be associated with higher job stress perceived and that role overload could be an important factor to consider as a source of stress in females.Key Words: job stress, physical activity, administrative, gender, age, seniority

Keywords: job stress, physical activity, clerks, gender, age, seniority

\section{Introducción}

En la actualidad, el aumento de la exigencia en el entorno laboral hace prácticamente inevitable la presencia de presión en el trabajo. Lo anterior deriva en la aparición de potenciales niveles de estrés laboral debido a una serie de trasformaciones a nivel económico, tecnológico y social que han provocado un impacto sobre las condiciones de los trabajadores y han generado una mayor vulnerabilidad hacia el estrés (Brannon y Feist, 2010; Marulanda, 2007).

Los efectos de tal patología se ven reflejados en problemas a nivel de salud mental y el deterioro en la salud física, peligrosos no solo para la salud y seguridad de cada trabajador, sino también para el buen funcionamiento de las entidades que se ven afectadas (Organización Mundial de la Salud [OMS], 2004). De esta manera, la importancia de la salud física y su relación con los índices de estrés laboral han marcado una estrecha relación con factores de realización personal, productividad y bienestar en el contexto laboral (Ranchal y Vaquero, 2008).

Diversas variables tienen una relación sobre diversos niveles de estrés en los contextos del ser humano, siendo las mujeres más afectadas ante ello debido a la multiplicidad de roles que cumplen como el ser madres, esposas, trabajadoras y ser miembros activos de una sociedad. Esta situación genera cambios constantes y permanentes tensiones que se trasladan de un contexto al otro (González, 2006; Oblitas, 2006; Straub, 2007; Weinberg y Gould, 2007). Factores como el agotamiento físico y mental, el doble rol laboral, la inseguridad laboral, las menores posibilidades de desarrollo laboral y la falta integración social constituyen algunos de los principales factores de riesgo que tienen un rango de afectación mayor en comparación con los hombres (Aranda, 2006; Catalina,Corrales,Ibermutuamur,Cortés y Gelpi, 2008; Pavlakis et al., 2010; Norlund et al., 2010).

También, ciertos estados civiles constituyen una variable generadora de estrés laboral, dentro de las cuales se puede mencionar que las separaciones o divorcios constituyen un suceso vital (eventos de tipo social que demandan en cada individuo realizar ciertos ajustes sobre las situaciones habituales) y son desencadenantes de estrés crónico cuando se manifiestan en 
periodos de relativa prolongación (Sandín, 2003). Percepciones de falta de apoyo social son percibidas en mayor frecuencia en mujeres jefas de hogar (Landero y González, 2011), pues las rupturas conyugales o divorcios constituyen uno de los eventos más estresantes y que en muchas ocasiones genera cambios en la red social reduciendo la densidad y duración de los lazos sociales. Por el contrario, el apoyo social recibido por el cónyuge se relaciona positivamente con la calidad de vida y con los menores niveles de estrés, ya que este es percibido como un mecanismo amortiguador (Garmendia et al., 2008; Haber et al., 2007; Suk y Sukhee, 2011).

La edad como variable relacionada con la percepción del estrés ha mostrado la tendencia en la cual los trabajadores más jóvenes y de menor experiencia presentan niveles más elevados de estrés laboral que las personas con mayor edad. Lo anterior está asociado a que las personas con mayor edad y experiencia laboral poseen mejores estrategias de afrontamiento para enfrentar factores generadores de estrés de su entorno (Weinberg y Gould, 2007). No obstante, la evidencia científica muestra datos muy contradictorios en los que los trabajadores, conforme incrementan su edad, muestran mayores índices de estrés laboral (Ahola et al., 2008). Ante ello, saltan a la luz posibles afirmaciones que manifiestan que durante la vida laboral, familiar y social se presentan una serie de aumentos y disminuciones en la acumulación de la carga total de situaciones generadoras de estrés en diferentes periodos de la vida; además, la época actual se ve marcada por las crecientes demandas en cuanto a competitividad que conllevan a un aprendizaje continuo y una variabilidad de horarios de trabajo, lo que genera una serie de cambios y alteraciones (síntomas de insomnio, agotamiento emocional, despersonalización) en el ámbito laboral a lo largo de la vida del trabajador (Gencay y Gencay, 2011; Hwan-Cheol et al., 2011; Pavlakis et al., 2010).

Asociado a lo anterior, diversos son los predictores desencadenantes de estrés laboral donde la frecuencia constante en su manifestación se ha relacionado positivamente $(\mathrm{p}=0.01)$ con altos niveles de presión en el trabajo y falta de apoyo social, reflejados en estadios de ansiedad y malestares subjetivos en salud (Berg et al., 2006; Sterud et al., 2011). De igual forma, la falta de organización en aspectos como la sobrecarga de trabajo, insuficiencia de recursos, la falta de comunicación, dinámicas de trabajo y estilos de gestión, entre otros, emergen como causas generadoras de estrés (Assadi, 2003).

Reportes en personal de salud (soporte médico) manifiestan una mayor frecuencia de ocurrencia de eventos generadores de estrés (presión en el trabajo, factores organizacionales) debido a la sucesión de eventos similares presentados de manera repetitiva $(\mathrm{p}<0.05)$, mientras que otros profesionales del mismo sector reportaron mayores niveles $(p<0.05)$ de intensidad de estrés en todas las dimensiones (presión en el trabajo, factores organizacionales), ligados a la atención de los pacientes y labores administrativas (Lua y Imilia, 2011).

Torres et al. (2010) reportaron, en una muestra de profesores, que la falta de apoyo social de las entidades organizativas y la presión en el trabajo surgen como los predictores de mayor influencia (58 \% y $64 \%$, respectivamente), siendo la cantidad de horas trabajas por semana una de las principales razones reportadas como fuente de estrés. 
Por otro lado, el nivel de actividad física o ejercicio físico realizado por un individuo es un factor de una estrecha relación con las manifestaciones de estrés en los diversos contextos de su vida (Oblitas, 2006; Straub, 2007). La práctica regular de ejercicio genera una serie de amortiguadores en el organismo (Brannon y Feist, 2010; Marks et al. 2008) con un gran efecto analgésico y relajante, así como una mayor liberación neurotransmisora, que permiten al individuo una mayor adaptación al estrés. Algunos estudios reportan que las personas que realizan ejercicio físico con mayor frecuencia semanal manifiestan índices menores de estrés percibido y mayor sensación de salud en relación con las personas que optan por estilos de vida más hipocinéticos (Jiménez et al., 2008); de igual manera, cuanta mayor actividad física se realice y mayor sea su intensidad, menores son los niveles de estrés y las manifestaciones psicosomáticas (Remor y Pérez-Llantada, 2007).

Tales efectos beneficios de la actividad física en las personas abarcan una variedad de etapas en el desarrollo humano. Portolés y González (2016), dentro de sus hallazgos, observaron percepciones menores en relación con el agotamiento emocional y mayor percepción de eficacia académica percibida en estudiantes de secundaria que realizaban actividad física entre los 30 a 60 minutos. De igual forma, la actividad física regular ha mostrado en otras poblaciones beneficios marcados en su salud mental, tal como sucede con lo plasmado por Orozco et al. (2016), quienes mostraron en un grupo de mujeres adultas mayores una mayor satisfacción hacia las actividades de la vida cotidiana y escasos niveles de ansiedad y depresión gracias a la práctica regular de Tai Chi como medio generador de actividad física. A nivel laboral, los trabajadores físicamente más activos muestran mejor percepción de sentirse más saludables, lo cual se ve reflejado en menores índices de estrés, mayores capacidades de productividad, incremento en los niveles de bienestar y satisfacción laboral (De Miguel et al., 2011; Viana et al., 2010).

A fin de estudiar las variables anteriormente descritas, el propósito del presente artículo es comparar los predictores del estrés laboral con los niveles de actividad física, la edad, la antigüedad laboral y el género en los empleados administrativos del sector público costarricense.

\section{Metodología}

La investigación se desarrolló bajo una línea de estudio transversal donde se recolectó una serie de información asociada a variables de tipo independiente y dependiente, en un momento determinado del tiempo y sobre el cual no existió una continuidad en la recolección de la información en el eje del tiempo siguiente. Las variables tratadas no se vieron manipuladas por la aplicación de un tratamiento determinado y el fin primordial ha sido describir una serie de comportamientos por medio de la aplicación de análisis de estadística tanto descriptiva como inferencial, por medio de la recolección de datos en entrevistas digitales respondidas por el grupo de participantes tomados en consideración (Heinemann, 2003; Hernández, 2007). 


\section{Participantes}

La muestra fue intencionada y estuvo compuesta por 395 sujetos del área administrativa de las áreas de recursos humanos, informática, financiero contable, proveeduría, bienes y servicios del MAG y pertenecientes a las oficinas de: Servicio Fitosanitario del Estado, Servicio Nacional de Salud Animal y oficinas centrales del MAG. Sobre su composición, un 52,7 \% de la muestra fue representada por el sexo masculino y el restante $47,3 \%$ por el sexo femenino. La edad promedio de los hombres fue de $49 \pm 9.84$ años y la de las mujeres de $45 \pm 11.54$ años. Según su estado civil, un $59 \%$ se identificó como casado, un 23,5 \% como soltero, 9,4 \% como divorciado, $6,8 \%$ como unión libre y un $1,3 \%$ como viudo.

En lo que respecta a la variable de años trabajados, un 43,5\% cuenta con 15 años o menos de laborar en dicha institución, un 36,5\% cuenta con un rango entre 16 a 30 años de tiempo laboral y un $20 \%$ con más de 30 años de laborar en la institución.

\section{Instrumentos}

Para la obtención de las variables predictoras se aplicó un cuestionario sociodemográfico con los siguientes datos: edad, género, tiempo laboral y estado civil. Para la evaluación de los predictores del estrés laboral se utilizó el cuestionario Job Stress Survey (JSS), versión cubana, y para la determinación del nivel de actividad física se utilizó el cuestionario internacional IPAQ (2005), versión corta.

El cuestionario Job Stress Survey (JSS) fue adaptado a la población costarricense de la versión cubana realizada por Marrero et al. (2011). El objetivo de este cuestionario es medir la intensidad (la cantidad de energía necesaria para hacerle frente a dichas situaciones) y frecuencia de los predictores de estrés laboral. El cuestionario se divide en dos dimensiones: intensidad de los factores estresantes (JSS Intensidad) y frecuencia con que ocurren los factores estresantes (JSS Frecuencia). Cada escala está constituida por 30 ítems, en una escala tipo Likert de 9 puntos (1 baja hasta 9 alto) que representa una serie de eventos relacionados con el trabajo y que dan origen a otras cuatro subescalas: presión de trabajo, falta de apoyo social, factores organizacionales y falta de realización personal. Para la escala JSS de intensidad del estrés, su objetivo fue valorar el grado de estrés que genera cada ítem tomando en consideración la cantidad de energía y tiempo que necesita para realizar dicho evento. Los análisis de confiabilidad realizados para las cuatro subescalas en la población costarricense ( $\alpha$ de Cronbach), se detallan a continuación: Presión de trabajo $(\mathrm{PTI})=0.74$, Falta de apoyo social $(\mathrm{FAI})=0.64$, Factores organizacionales $(\mathrm{FOI})=0.79$ y Falta de realización personal $(F R P I)=0.70$. Por su parte, la escala JSS frecuencia del estrés tiene como objetivo la determinación de con qué frecuencia se han presentado tales eventos en los últimos seis meses y presenta una puntuación de 0 a 9 (cero si no se presentó el evento y el número 9 si lo ha tenido nueve días o más durante los últimos seis meses). Los resultados de su confiabilidad en la población costarricense ( $\alpha$ de Cronbach), para cada una de sus subescalas 
se detallan a continuación: Presión de trabajo $(\mathrm{PTF})=0.80$, Falta de apoyo social $(\mathrm{FAF})=0.60$, Factores organizacionales $(\mathrm{FOF})=0.75$ y Falta de realización personal $(\mathrm{FRPF})=0.77$.

En cuanto al cuestionario IPAQ Corto - Nivel de actividad física, este se encuentra constituido por 7 preguntas generadoras cuyo objetivo es obtener información sobre el nivel de actividad física relacionada con la salud. Este cuestionario es de tipo autoadministrado y puede ser utilizado en jóvenes y adultos con edades entre los 15 a 69 años; además, según estudios realizados en 12 países, se sugiere que posee propiedades transculturales aceptables de medición en relación con la confiabilidad (Spearman's rho $=0.80)$ y validez (Spearman's rho $=0.30$ ) (Hallal, et al., 2010; IPAQ, 2005). Para determinar el nivel de actividad física en cada participante, este se basa en los METS para las actividades semanales de caminata, actividad física moderada e intensa; además, mediante la determinación del gasto metabólico para cada una de las actividades, se procede a la determinación del total de actividad física semanal (IPAQ, 2005): a) caminata MET - minutos $/$ semana $=3.3 *$ minutos caminata $*$ días de caminata; b) Moderado MET - minutos $/$ semana $=4.0 *$ minutos actividad moderada $*$ días de actividad moderada; y c) Vigorosa MET - minutos/semana $=8.0 *$ minutos actividad vigorosa * días de actividad vigorosa. Mediante la obtención del gasto metabólico para cada una de las categorías según criterios de categorización, se procede a clasificar a los individuos en personas que realizan actividad física vigorosa, moderada, o bien, personas que realizan poca actividad física (Serón et al., 2010).

Los criterios para la determinación del tipo de actividad física que desempeña cada sujeto son los siguientes:

1. Nivel de actividad física alto: a) Actividad física vigorosa al menos tres veces por semana y un nivel de actividad física total de al menos 1500 METs/ minuto por semana. b) Siete días o más de cualquier combinación de actividad de caminata, actividad física moderada o vigorosa y un nivel de actividad física total de $3000 \mathrm{METs} /$ minuto por semana.

2. Nivel de actividad física moderado: a) 3 o más días de AF vigorosa y/o caminar al menos 30 minutos al día. b) 5 o más días de AF moderada o caminar al menos 30 minutos al día. c) 5 o más días de cualquier combinación entre andar, AF moderada o vigorosa y una "actividad física total" mínima de $600 \mathrm{METs} /$ minutos por semana.

3. Nivel de actividad física bajo: a) Todas aquellas actividades que no se incluyen en los niveles alto y moderado.

\section{Procedimiento}

Para la obtención de los resultados se procedió primeramente con la coordinación del departamento de recursos humanos, específicamente con el director de recursos humanos del MAG en su sede central, con el cual se coordinó un consentimiento informado para la consecución 
de la población meta del estudio y la colaboración en cuanto a la metodología de aplicación de los cuestionarios de investigación. Esta consistió en la aplicación de cuestionarios electrónicos diseñados por el evaluador principal mediante el software gratuito freeonlinesurveys y cuyo procedimiento ha sido utilizado en otras investigaciones (Forneris et al., 2012; Lauber et al., 2005).

Una vez obtenida la población meta y la aprobación por parte del MAG, se procede a confeccionar el cuestionario, realizar pruebas internas y elaborar una prueba piloto. Para finalizar, se envía el cuestionario en línea desde la dirección de recursos humanos del MAG hacia todos los funcionarios administrativos de las tres oficinas en las cuales se realizó el estudio. Para la recolección de los datos se tomaron todos los cuestionarios completados y reenviados entre las fechas comprendidas del 01 de octubre del 2012 hasta el 26 de octubre del 2012.

\section{Análisis estadístico}

Se aplicó estadística descriptiva y estadística inferencial con análisis de varianza factorial para la determinación de la relación entre las variables predictoras y dependientes. Todos los análisis se realización con el paquete estadístico SPSS 21.0 para Microsoft Windows.

\section{Resultados}

Los resultados del análisis del cuestionario Job Stress Survey (JSS) para la dimensión intensidad (definida como la energía necesaria para poder afrontar situaciones generadoras de estrés) se muestra a continuación:

Tabla 1

Comparación en el puntaje obtenido en los predictores de estrés laboral "Presión en el trabajo" y "Falta de organización” según el género.

\begin{tabular}{|c|c|c|}
\hline & \multicolumn{2}{|c|}{ Intensidad de estrés generado } \\
\hline & Presión en el trabajo & Factores organizacionales \\
\hline Femenino & $43.3^{*}$ & $52.27 * *$ \\
\hline Masculino & 39.36 & 46.83 \\
\hline
\end{tabular}

$* p=.042 ; * * p=.010$, entre géneros 
En la tabla 1, se observa una puntuación mayor y significativa en la percepción de los funcionarios de género femenino en relación con la energía demandada para afrontar situaciones de estrés relacionadas con la presión en el trabajo $(\mathrm{F}=2.037 ; \mathrm{p}=.042)$ y los factores de tipo organizacionales $(\mathrm{F}$ $=2.574 ; \mathrm{p}=.010$ ), en relación con la percepción de los funcionarios de género masculino.

Tabla 2

Comparación en el puntaje obtenido en los predictores de estrés laboral "Presión en el trabajo" y "Falta de organización" según el género.

\begin{tabular}{lccc}
\hline & \multicolumn{3}{c}{ Frecuencia de ocurrencia } \\
\hline & Presión en el trabajo & Falta de apoyo social & Factores organizacionales \\
\cline { 2 - 4 } Femenino & $48.8^{*}$ & $21.42^{* *}$ & $53.48^{* * *}$ \\
Masculino & 41.4 & 18.67 & 45.79 \\
\hline
\end{tabular}

$* p=.001 ; * * p=.011 ; * * * p=.001$, entre géneros

La tabla 2 muestra una puntuación mayor y significativa en la percepción de los funcionarios de género femenino en relación con la frecuencia en la que se manifiestan en los últimos seis meses situaciones de estrés relacionadas con la presión en el trabajo $(\mathrm{F}=3.506 ; \mathrm{p}=$ $.001)$; falta de apoyo social $(\mathrm{F}=2.563 ; \mathrm{p}=.011)$ y factores de tipo organizacionales $(\mathrm{F}=3.364$; $\mathrm{p}=.001)$, en relación con la percepción de los funcionarios de género masculino.

Tabla 3

Comparación en el puntaje obtenido para el predictor de estrés laboral "Falta de realización personal", perteneciente a la escala relacionada con la frecuencia de ocurrencia en los últimos seis meses, según el estado civil en una población de funcionarios administrativos.

\begin{tabular}{lccccc}
\hline & \multicolumn{5}{c}{ Estado civil } \\
\hline \multirow{2}{*}{$\begin{array}{c}\text { Falta de realización } \\
\text { personal* }\end{array}$} & Soltero & Casado** $^{*}$ & Unión libre & Divorciado** & Viudo \\
\cline { 2 - 5 } & 14.62 & 12.31 & 12.63 & 17.46 & 14.6 \\
\hline$p=.009$, entre los estados civiles; $* * p=.008$, entre géneros casado y divorciado.
\end{tabular}

En la tabla 3 se puede observar que existen diferencias significativas $(F=3.343 ; p=.009)$ entre los grupos evaluados según el estado civil y su relación con la frecuencia de manifestación del predictor de estrés laboral asociado a la falta de realización personal en los últimos seis meses. Análisis posteriores (post-hoc de tukey) evidencian diferencias significativas $(\mathrm{p}=.008)$ específicamente entre los trabajadores con un estado civil "divorciado", quienes manifiestan una mayor falta de realización personal en comparación con los trabajadores cuyo estado civil es "casado". 
Tabla 4

Comparación en el puntaje obtenido para los predictores de estrés laboral pertenecientes a la escala relacionada con la intensidad o energía demandada, según la antigüedad laboral en una población de funcionarios administrativos.

\begin{tabular}{lcccccccc}
\hline & \multicolumn{4}{c}{ Intensidad } & \multicolumn{3}{c}{ Frecuencia } \\
\cline { 2 - 9 } & PT & FA & FRP & FO & PT & FA & FRP & FO \\
\cline { 2 - 9 } 0 a 15años* & 42.51 & 20.01 & 13.81 & 50.08 & 45.01 & 19.74 & 13.25 & 48.18 \\
16 a 30 años* & 41.18 & 19.63 & 12.61 & 49.85 & 45.42 & 20.06 & 13.23 & 50.12 \\
Más de 30 años* & 38.51 & 19.34 & 13.19 & 47.14 & 43.75 & 20.32 & 13.97 & 50.89 \\
\hline
\end{tabular}

PT $=$ Presión en el trabajo; FA = Factores organizacionales; FRP = Falta de realización personal; $F O=$

Factores organizacionales

$* P>0.05$, para los distintos predictores de estrés laboral entre los rangos de tiempo laboral.

En la tabla 4 se observa la asociación entre cada uno de los predictores del estrés laboral relacionados con la energía demanda para afrontarlos (intensidad) y la frecuencia con la cual se ha suscitado en los últimos seis meses, según distintos rangos de antigüedad laboral. Mediante un análisis de varianza, no se encontraron diferencias estadísticamente significativas en los predictores "Presión en el trabajo", "Falta de apoyo en el trabajo", "Falta de organización" y "Falta de realización personal", según la antigüedad laboral; lo cual indica que no existen importantes variaciones en la percepción entre los diversos predictores del estrés laboral relacionados con la utilización de energía necesaria para afrontarlos y su frecuencia de manifestación.

Tabla 5

Clasificación del nivel de actividad física a nivel general y según el género en un grupo de funcionarios administrativos.

\begin{tabular}{cccc}
\hline & & Nivel de actividad física & \\
\hline & Bajo & Medio & Alto \\
\cline { 2 - 4 } Masculino & 48.6 & 44.2 & $68.2^{*}$ \\
Femenino & 51.4 & 45.05 & 31.8 \\
General & $72.4^{* *}$ & 10.9 & 16.7 \\
\hline
\end{tabular}

$* p=.007$, entre genero para el nivel de actividad fisico “Alto”.

$* * p=.002$, entre los niveles de actividad física de forma general. 
En la tabla 5 se observan los valores referentes a los niveles de actividad física entre los funcionarios administrativos donde se observa, de forma significativa $(p=.002)$, un mayor porcentaje de funcionarios con bajos niveles de actividad física. Por su parte, al realizar la comparación entre géneros, se observa menores de niveles de actividad en los funcionarios de género femenino, pero de manera significativa $(\mathrm{p}=.007)$ se observa que los funcionarios masculinos realizan una mayor cantidad de actividad física de un nivel alto en comparación con las funcionarias.

Tabla 6

Comparación entre la percepción (porcentaje) de la energía demanda (intensidad) y la frecuencia de ocurrencia de los factores generadores de estrés laboral, según los distintos niveles de realización de actividad física en un grupo de funcionarios administrativos.

\begin{tabular}{ccc}
\hline & \multicolumn{2}{c}{ Factores organizacionales } \\
\hline & Intensidad* & Frecuencia** \\
\cline { 2 - 3 } Bajo & 51.23 & 51.41 \\
Moderado & 45.05 & 45.26 \\
Alto & 44.36 & 43.36 \\
\hline
\end{tabular}

${ }^{*} p=.021$, entre los niveles de actividad fisica según la energía demandada.

** $p=.019$, entre los niveles de actividad fisica según la frecuencia de ocurrencia.

En la tabla 6 se pueden observar diferencias significativas $(p=.021)$ entre los niveles de actividad física en relación con el factor generador de estrés laboral denominado "factores organizacionales", según la energía demandada para hacerle frente (intensidad). Mediante un análisis posterior (posthoc de Tukey), se encontró que dichas diferencias de forma significativas se presentaron $(\mathrm{p}=.045)$ entre los funcionarios que poseen bajas niveles de actividad física (mayor afectación) en comparación con los funcionarios que realizan mayor actividad física de una forma intensa.

De igual forma, en la misma tabla, se pueden observar diferencias significativas $(p=.019)$, entre los niveles de actividad física en relación con el factor generador de estrés igualmente asociado a "factores organizacionales", pero referente a la frecuencia de ocurrencia en los últimos seis meses. Mediante un análisis posterior (post-hoc de Tukey), se encontró que dichas diferencias de forma significativas se presentaron $(p=.032)$ entre los funcionarios que poseen bajos niveles de actividad física (mayor frecuencia de presencia) en comparación con los funcionarios que realizan actividad física de una forma intensa.

\section{Discusión}

La sensación de estrés en el contexto del trabajo dependerá en gran medida de la valoración subjetiva que cada empleado realice sobre una circunstancia en particular y cuando esta genere, 
en cierta medida, un desajuste en su contexto actual. Tales estresores pueden ser valorados y analizados desde varias dimensiones, como son la frecuencia de ocurrencia e intensidad demandada para su atención y resolución (Berg et al., 2006; Campo et., 2009; Gómez y Escobar, 2006; González et al., 2011; Oblitas, 2006; Peiró y Rodríguez, 2008). Ante ello, el propósito del presente artículo fue comparar los predictores del estrés laboral con los niveles de actividad física, la edad, la antigüedad laboral y el género en un grupo de funcionarios administrativos del sector público costarricense.

Al comparar el género con los predictores del estrés laboral, las funcionarias mostraron puntaciones promedio mayores en relación con los funcionarios, tanto para los predictores asociados con la intensidad y frecuencia de ocurrencia relacionados con la presión en el trabajo y la falta de organización. De manera similar, Berg et al. (2006), en policías noruegos, encontraron valores elevados para la frecuencia e intensidad del predictor asociado a la presión en el trabajo, mientras que en las mujeres se observaron valores superiores en la intensidad en el predictor de presión en el trabajo. Asimismo, Sterud et al. (2011) y Lua e Imilia (2011) encontraron, en personal de la salud, puntuaciones altas para los predictores relacionados con la presión en el trabajo y la falta de organización, de manera que se relacionaron significativamente con los niveles de estrés. Del mismo modo, Assadi et al. (2003), en organizaciones relacionadas con la educación física, encontraron altos niveles de estrés en relación con el predictor asociado a la falta de organización en tales empresas, principalmente en la percepción de la intensidad de este. Igualmente, Mack y McGee (2001) observaron en una serie de pymes altas puntaciones en el predictor del estrés concerniente a la falta de organización; por su parte, Torres et al. (2010) reportaron, en un grupo de profesores de secundaria, como principal predictor del estrés laboral la presión en el trabajo, aspecto en el que las mujeres presentaron niveles superiores en relación con los hombres. Por último, Aranda (2006) reporta, en un grupo de médicos familiares, una mayor presión laboral en las mujeres, lo cual las vuelve más vulnerables a agotarse física y mentalmente.

Ante esta situación, una mayor afectación sobre las mujeres puede estar asociada a un incremento en la sobrecarga de funciones debido a la multiplicidad de roles, generando con ello cambios constantes y tensiones permanentes que se trasladan de un contexto al otro, en comparación con los hombres (Catalina et al., 2008; González, 2006; Norlund et al., 2010; Oblitas, 2006; Pavlakis et al., 2010; Ranchal y Vaquero, 2008; Straub, 2007). Además, aspectos como la dinámica de trabajo, la deficiente estructura organizativa, la falta de cooperación y la complejidad de tareas que se presentan muy a menudo de manera similar y repetitiva, tienen una importante influencia sobre la intensidad y frecuencia de los predictores de estrés laboral (Assadi, 2003; Torres et al., 2010).

Al analizar el estado civil, en los funcionarios divorciados se encontraron puntaciones superiores, en relación con los funcionarios cuyo estado civil es casado, en la frecuencia de ocurrencia en la percepción de una mayor falta de apoyo social, mostrando la posibilidad de que los funcionarios divorciados frecuentemente experimentan como fuente de estrés laboral la falta de apoyo social. Tales resultados concuerdan con Borda et al. (2007), quienes apuntaron que la mayoría de las personas sin cónyuge experimentaron una mayor falta de apoyo social 
como fuente de estrés laboral en comparación con las personas casadas. Por su parte, Landero y González (2011) encontraron que las familias de conformación monoparantales poseen una mayor percepción de falta de apoyo social, en comparación con aquellos núcleos familiares de conformación biparentales. Ante ello, salta a luz que los trabajadores divorciados son más propensos a percibir una mayor falta de apoyo social que los casados. En este sentido, Sandín (2003) menciona que las separaciones o divorcios constituyen un importante suceso vital que genera efectos directos sobre la salud física y mental, ocasionando durante periodos relativamente prolongados una serie de sucesos cotidianos no esperados que pueden desencadenar en estados de estrés crónico. Asimismo, los estados civiles de divorciado, viudo o soltero tienen la probabilidad de percibir con mayor frecuencia la falta de apoyo social debido a la sensación de una falta de afiliación que constituye un elemento esencial en el desarrollo personal del individuo (Hernández et al., 2011). Por su parte, aquellas personas casadas suelen poseer una mayor sensación de autorrealización (Arias y Jiménez, 2013) y en la que el apoyo del cónyuge simboliza una de las principales fuentes de apoyo social (Landero y González, 2011).

Por su parte, la antigüedad laboral y los diversos factores generadores de estrés laboral permiten analizar que, conforme aumenta la experiencia laboral, existe una ligera disminución no significativa en el puntaje reportado concerniente a la intensidad generada y la frecuencia de ocurrencia de los predictores de estrés por parte de los funcionarios; no obstante, esto concuerda en parte con lo mencionado por Weinberg y Gould (2007), quienes estipulan que las personas con mayor experiencia laboral presentan niveles menores de estrés laboral, en comparación con los trabajadores con una experiencia laboral menor, principalmente por que cuentan con estrategias de afrontamiento más efectivas. Pavlakis et al. (2010) reportan datos similares en profesionales del área de salud, en los que el aumento en la antigüedad laboral se asocia a una menor expresión de síntomas de estrés. Sin embargo, tales no significancias y variaciones a lo largo de la vida laboral de un trabajador en sus manifestaciones de estrés concuerdan con lo mencionado por Ahola et al. (2008), quienes, tras un análisis con trabajadores de distintos rangos de experiencia laboral y puestos laborales, determinaron que a lo largo de la vida laboral se presentan aumentos y disminuciones en la acumulación de la carga total de situaciones generadoras de estrés. Además, Ranchal y Vaquero (2008) argumentan que las crecientes demandas de la época actual requieren un aprendizaje continuo y competencias que generan cambios a lo largo de la vida laboral de las personas, ocasionando con ello estadios de estrés a lo largo de la vida laboral de las personas.

En cuanto a los niveles de actividad física entre los géneros, se observó que las funcionarias administrativas muestran niveles de actividad física menores en relación con los hombres, permitiendo suponer que estos últimos son físicamente más activos en comparación con las mujeres para los niveles de actividad física moderado y vigoroso. Estos resultados se asemejan a los ya encontrados por otros autores (Hawkins et al., 2009; Trinh et al., 2008) y siguen la misma tendencia encontrada en poblaciones de otras latitudes, como sucede en el caso de la población Australiana, donde las mujeres presentan mayores niveles de inactividad física, (Victorian Health Promotion Foundation; 2010) y en el caso Canadá, en donde el porcentaje de hombres físicamente 
activos es superior en relación con las mujeres (Human Resources and Skills Development Canada, 2011). Tales diferencias encontradas entre géneros pueden ser atribuibles a diversos factores, desde la estigmatización social hacia el ejercicio físico y deporte (Loland, 2000; Moreno et al., 2006), que trae consigo el abandono de dichas prácticas por parte de las mujeres a tempranas edades y que repercute en edades posteriores. De igual forma, el tiempo dedicado a las labores del hogar y cuidado de la familia parece ser una fuente importante que limita el tiempo libre (Loland, 2000).

Los niveles de actividad física y la interacción con los distintos predictores de estrés laboral mostraron asociaciones entre aquellas personas cuyo nivel de actividad física fue vigoroso y la menor percepción a la intensidad generada, junto con una menor frecuencia con que se han presentado de los factores generadores de estrés; es decir, la energía que les demanda el poder afrontar situaciones relacionadas con problemas organizacionales en contraste con las personas cuyo nivel de actividad física es bajo. Resultados similares han sido reportados por Jiménez et al. (2008), quienes indicaron que aquellos sujetos que realizaron de manera regular ejercicio físico presentaron mayor bienestar psicológico y menores niveles de estrés percibido. Igualmente, Remor y Pérez-Llantada (2007) concuerdan en que a una mayor frecuencia e intensidad de la actividad física, menores son las percepciones de estrés psicológico, ya que cuanta más actividad física realizada y, cuanto mayor sea su intensidad, menores son los niveles de estrés en comparación con las personas sedentarias. De igual forma, una mayor continuidad de estilos de vida más activos genera mayores sensaciones salud y adecuadas estrategias de afrontamiento (De Miguel et al., 2011); también, tales niveles inferiores de percepción de estrés en personas cuyo nivel de actividad física es mayor poseen un trasfondo fisiológico, ya que existe una mayor facilitación de transmisión en el torrente sanguíneo a nivel cerebral de noradrenalina, serotonina y dopamina, además de incrementar los niveles de endorfinas producto de la práctica continua de ejercicio físico, generando a mediano plazo cambios permanentes en el cerebro relacionados con mayores estados de ánimo, optimismo y bienestar (Brannon y Feist, 2010; Marks et al., 2008).

\section{Conclusiones}

Las funcionarias parecen manifestar un mayor degaste de energía y una mayor frecuencia, en los últimos seis meses, de situaciones generadoras de estrés laboral asociadas a una mayor presión en el trabajo, la falta de organización y la falta de apoyo social, en comparación con los hombres.

Las mujeres presentan un nivel de actividad física mucho menor en relación con los hombres, tanto para todas aquellas actividades de moderada y vigorosa intensidad. Estas manifestaciones pueden sugerir que la escasez de tiempo, la sobrecarga de funciones, y sin dejar de lado la posible estigmatización social hacia el ejercicio, son limitantes de un estilo de vida hipocinético.

Los funcionarios cuyo estado civil es divorciado experimentan con mayor frecuencia, en comparación con los funcionarios casados, la falta de realización personal como fuente generadora de estrés en el trabajo, dando pie a que dicho estado civil se considere un suceso 
vital negativo que requiere ciertas modificaciones en sus vidas; además, el cónyuge constituye una de las principales redes de apoyo en las parejas.

La antigüedad laboral de los funcionarios y su relación con los distintos predictores del estrés laboral no refleja con claridad una tendencia dominante con respecto a la disminución o bien aumento en la percepción de las fuentes de estrés, tanto en la demanda de energía y su frecuencia con que se presenten. Esto podría estar asociado al hecho de que a lo largo de la vida laboral se presentan en momentos diversos, situaciones de pueden suscitar estadios de estrés, producto del proceso de modernización y globalización del sector laboral.

A mayores niveles de actividad física, se observa la tendencia de una menor percepción de energía necesaria y una menor frecuencia de percepción de aparición de factores generadores de estrés laboral relacionados con la presión en el trabajo. De igual manera, aquellos trabajadores cuyo nivel de actividad física es vigoroso perciben en menor medida los factores generadores de estrés en comparación con los niveles de actividad física menores.

\section{Referencias bibliográficas}

Ahola, K., Honkonen, T., Virtanen, M., Aromaa, A. \& Lönnqvist, J. (2008). Burnout in relation to age in the adult working population. Journal of Occupational Health, 50, 362-365. Recuperado de: http://dx.doi.org/10.1539/joh.M8002

Aranda, C. (2006). Diferencias por sexo, síndrome de burnout y manifestaciones clínicas, en los médicos familiares de dos instituciones de salud, Guadalajara, México. Revista costarricense de salud pública, 15(19). Recuperado de http://www.scielo.sa.cr/pdf/rcsp/ v15n29/3316.pdf

Arias, W.L., y Jiménez, N.A. (2013). Síndrome de burnout en docentes de Educación Básica Regular de Arequipa. Educación, 22(42), 53 - 76. Recuperado de http://revistas.pucp.edu. pe/index.php/educacion/article/view/5291/5288

Assadi, H. (2003). Evaluation of job stress factors (organizational and managerial) among heads of physical education organizations. Acta universitatis palackianae olomucensis gymnica, 33(1), 47-55. Recuperado de: http://publib.upol.cz/ obd/fulltext/Gymnica33-1/gymnica7.pdf

Berg, A.M., Hem, E., Lau, B., \& Ekeberg, Ø. (2006). An exploration of job stress and health in the Norwegian police service: a cross sectional study. Journal of Occupational Medicine and Toxicology, 1(26). http://dx.doi.org/10.1186/1745-6673-1-26

Borda, M., Navarro, E., Aun-Aun, E., Berdejo, H., Racedo Rolón, K. y Ruiz, J. (2007). Síndrome de Burnout en estudiantes de internado del Hospital Universidad del Norte. Salud Uninorte, 23(1), 43-51. Recuperado de: http://www.scielo.org.co/pdf/sun/v23n1/v23n1a06.pdf 
Brannon, L., \& Feist, J. (2010). Health Psychology. An Introducction to behavior and health [Psicología de la Salud. Una Introducción con el comportamiento y la salud] (7ª ED.). Belmont, Estados Unidos de America: Wadsworth.

Catalina, C., Corrales, H., Ibermutuamur, Cortés, M.V. y Gelpi, J.A. (2008). Las diferencias de género en el nivel de exposición a riesgos psicosociales entre trabajadores/as de la comunidad autónoma de Madrid. Resultados preliminares del plan de investigación del estrés laboral de Ibermutuamur. Revista de psicología y psicopedagogía, 7(2), 185-200. Recuperado de: $\underline{\text { http://dialnet.unirioja.es/servlet/fichero articulo?codigo=2800135.pdf }}$

Campo, A., Bustos, G.J. y Romero, A. (2009). Consistencia interna y dimensionalidad de la Escala de Estrés Percibido (EEP-10 y EEP-14) en una muestra de universitarias de Bogotá, Colombia. Aquichan, 9(3), 271-280. Recuperado de: http://redalyc.uaemex.mx/src/inicio/ ArtPdfRed.jsp?iCve $=74112147007$

De Miguel, J.M., Schweiger, I., de las Mozas, O. y Hernández, J.M. (2011). Efecto del ejercicio físico en la productividad laboral y el bienestar. Revista de Psicología del Deporte, 20(2), 589-604. Recuperado de http://dd.uab.cat/pub/revpsidep/19885636v20n2/19885636v20n2p589.pdf

Forneris, T., Camiré, M., \& Trudel, P. (2012). The development of life skills and values in high school sport: Is there a gap between stakeholder's expectations and perceived experiences?. International Journal of Sport and Exercise Psychology. 10(1), 9-23. D http://dx.doi.org/1 $\underline{0.1080 / 1612197 X .2012 .645128}$

Garmendia, M.L., Alvarado, M.E., Montenegro, M. y Pino, P. (2008). Importancia del apoyo social en la permanencia de la abstinencia del consumo de drogas. Revista médica de Chile, 136(2), 169-178. Recuperado de: http://www.scielo.cl/pdf/rmc/v136n2/art05.pdf

Gencay, S. \& Gencay, O. A. (2011). Burnout among judo coaches in Turkey. Journal of Occupational Health, 53, 365-370. Recuperado de: http://joh.sanei.or.jp/pdf/E53/E53 5 09.pdf

Gómez, B. y Escobar, A. (2006). Estrés y sistema inmune. Revista mexicana de neurociencia, 7(1), 30-38. Recuperado de: http://www.neurologia.org.mx/portalweb/documentos/ articulos/Nm061-05.pdf

González, M.G. (2006). Estrés laboral, afrontamiento y sus consecuencias: el papel del género (Tesis no publicada de Doctorado en Psicología). Universidad de Valencia, Valencia, España. Recuperado de: http://www.uv.es/maglogon/GonzalezMorales2006TESIS.pdf

González, M., García, J. y Landero, R. (2011). El papel de la teoría transaccional del estrés en el desarrollo de la fibromialgia: un modelo de ecuaciones estructurales. Actas Españolas de Psiquiatría, 39(2), 81-87. Recuperado de: http://actaspsiquiatria.es/repositorio/13/70/ ESP/13-70-ESP-81-87-775273.pdf 
Hallal, P., Gómez, L.F., Parra, D., Lobelo, F., Mosquera, J., Florindo, A., Reis, R., Pratt, P., y Sarmiento, O. (2010). Lecciones aprendidas después de 10 Años del uso de IPAQ en Brasil y Colombia. Journal of Physical Activity and Health, 7(2), S259-S264. Recuperado de: http://www.rafapana.org/attachments/article/40/JPAH_IPAQ_es.pdf

Haber, M.G., Cohen, J.L., Lucas, T. Baltes, B.B. (2007). The relationship between selfreported received and perceived social support: A meta-analytic review. American Journal of Community Psychology, 39: 133-44. http://dx.doi.org/10.1007/s10464-007-9100-9

Hawkins, M.S., Storti, K.L., Richardson, C. R., King, W. C., Strath, S. J., Holleman, R.G., \& Kriska, A.M. (2009). Objectively measured physical activity of USA adults by sex, age, and racial/ethnic groups: a cross-sectional study. International Journal of Behavioral Nutrition and Physical Activity, 6(31). http://dx.doi.org/10.1186/1479-5868-6-31

Heinemann, K. (2003). Introducción a la metodología de la investigación empírica en las ciencias del deporte. Barcelona: Editorial Paidotribo.

Hernández Ávila, M. (2007). Epidemiología. Diseño y análisis de estudios. México D.F.: Editorial Médica Panamericana.

Hernández, J.T., Granados, P.A. y Carmona, J.J. (2011). Posibles indicadores de síndrome de Burnout, en 18 operarios de una distribuidora de GLP de la ciudad de Armenia. Revista científica electrónica de ciencias sociales, 20(7), 22-37. Recuperado de http://www. revistanegotium.org.ve/pdf/20/art2.pdf

Human Resources and Skills Development Canada. (2011). Indicators of Well-being in Canada. Health-Physical Activity. Recuperado de: http://www4.hrsdc.gc.ca/.3ndic.1t.4r@-eng.jsp?iid=8

Hwan-Cheol, K., Byeong-Kwon, K., Kyoung-Bok, M., Jin-Young, M., Sang-Hee, H. \& Shin-Goo, P. (2011). Association between job stress and insomnia in Korea workers. Journal of Occupational Health, 53, 164-174. Recuperado de http://joh.sanei.or.jp/pdf/E53/E53_3 02.pdf

IPAQ. (2005). Guidelines for Data Processing and Analysis of the International Physical Activity Questionnaire (IPAQ). Recuperado de www.ipaq.ki.se/scoring.pdf

Jiménez, M.G., Martínez, P., Miró, E. y Sánchez, A.I. (2008). Bienestar psicológico y hábitos saludables: ¿están asociados a la práctica de ejercicio físico? International Journal of Clinical and Health Psychology, 8(1), 185-202. Recuperado de: http://www.aepc.es/ijchp/ articulos_pdf/ijchp-272.pdf

Landero, R. y González, M. (2011). Apoyo social, estrés y autoestima en mujeres de familias monoparentales y biparentales. SUMMA psicología UST, 8(1), 29-36. Obtenido de https:// dialnet.unirioja.es/descarga/articulo/3738116.pdf 
Lauber, C., Ajdacic-Gross, V., Fritschi, N., Stulz, N. \& Rössler, W. (2005). Mental health literacy in an educational elite - an online survey among university students. Biomedical Central, 5(44). doi:10.1186/1471-2458-5-44.

Loland, N. W. (2000). The aging body: attitudes toward bodily appearance among physically active and inactive women and men of different. Journal Of Aging \& Physical Activity, 8(3), 197-213. Recuperado de: http://search.ebscohost.com/login. aspx?direct $=$ true $\& \mathrm{db}=\mathrm{s} 3 \mathrm{~h} \& \mathrm{AN}=\mathrm{SPHS}-656478 \&$ lang=es\&site=ehost-live

Lua, P.A. \& Imimia, I. (2011). Work-Related Stress Among Healthcare Providers of Various Sectors in Peninsular Malaysia. Malaysian Psychiatric Association, 1(9), Recuperado de: http://www.mjpsychiatry.org/index.php/mjp/article/viewFile/153/127

Mack, D.A. \& McGee, J.E. (2001). Occupational Stress and the Small Business Owner: The Role of Task Complexity and Social Support. Submitted to the USASBE/SBIDA - 2001 Conference Small Business Division. Recuperado de: http://citeseerx.ist.psu.edu/viewdoc/ $\underline{\text { download?doi }=10.11 .199 .3260 \& \mathrm{rep}=\text { rep1\&type }=\text { pdf }}$

Marrero, M.L., Rivero, E., Pastor, M.E., Fernández, C. y Vergara, A. (2011). Elaboración de la versión cubana del cuestionario JSS para la evaluación del estrés psicosocial laboral. Revista Cubana de Salud y Trabajo, 12(2), 9-18. Recuperado de: http://bvs.sld.cu/revistas/ rst/vol12_2 11/rst02211.pdf

Marks, D., Murray, M., Evans, B., Willig, C., Woodall, C. y Sykes, C. (2008). Psicología de la salud. Teoría, Investigación y práctica. Bogotá, Colombia: Manual Moderno.

Marulanda Ruiz, I. C. (2007). Estrés laboral enemigo silencioso de la salud mental y la satisfacción con la vida. Bogotá, Colombia: Uniandes. Recuperado de: http:// publicacionesfaciso.uniandes.edu.co/psi/Estr_s_Laboral.pdf

Moreno, J. A., Martínez, C., Alonso, N. (2006). Actitudes hacia la práctica físico-deportiva según el sexo del practicante. Revista Internacional de Ciencias del Deporte. 3(2), 20-43. http://dx.doi.org/10.5232/ricyde2006.00302

Norlund, S., Reuterwall, C., Höög, J., Lindahl, B., Janlert, U., \& Slunga Birgander, L. (2010). Burnout, working conditions and gender results from the northern Sweden MONICA Study. BMC Public Health,10(326). http://dx.doi.org/10.1186/1471-2458-10-326

Oblitas, L. (2006). Psicología de la Salud y calidad de vida (2 Ed.). Distrito Federal, México: Thomson.

Organización Mundial de la Salud. (2004). La organización del trabajo y estrés. Serie protección de la salud de los trabajadores. (3). Recuperado de: http://whqlibdoc.who.int/ pwh/2004/9243590472.pdf 
Orozco, G., Anaya, M., Santiago, J., y García, M. (2016). Cognición, actividades de la vida diaria y variables psicológicas mujeres adultas mayores practicantes de Tai Chi Chuan (Yang). Retos, 30, 222-225. Obtenido de http://recyt.fecyt.es/index.php/retos/article/ download $/ 50343 / 30784$

Pavlakis, A., Raftopoulos, V. \& Theodorou, M. (2010). Burnout syndrome in Cypriot physiotherapists: a national survey. BMC Health Services Research, (10)63. http://dx.doi. org/10.1186/1472-6963-10-63

Peiró, J.M, \& Rodríguez, I. (2008). Work stress, leadership and organizational health. Papeles del Psicólogo, 29(1), 68-82. Recuperado de http://www.papelesdelpsicologo.es/english/1540.pdf

Portolés, A., y González, J. (2016). Actividad física y niveles de burnout en alumnos de la E.S.O. Retos, 29, 95-99. Obtenido de http://recyt.fecyt.es/index.php/retos/article/ download $/ 39933 / 23470$

Ranchal , A. y Vaquero, M. (2008). Burnout, variables fisiológicas y antropométricas: Un estudio en el profesorado. Medicina y Seguridad del Trabajo, 54(210), 47-55. Recuperado de http://dx.doi.org/10.4321/s0465-546x2008000100007

Remor, E. y Pérez-Llantada, M.C. (2007). La Relación entre Niveles de la Actividad Física y la Experiencia de Estrés y de Síntomas de Malestar Físico. Revista Interamericana de Psicología/Interamerican Journal of Psychology, 41(3), 313-322. Recuperado de: http:// redalyc.uaemex.mx/src/inicio/ArtPdfRed.jsp?iCve $=28441306$

Sandín, B., (2003). El estrés: un análisis basado en el papel de los factores sociales. Revista Internacional de Psicología Clínica y de la Salud, 3(1), 141 - 157. Recuperado de http:// www.aepc.es/ijchp/articulos_pdf/ijchp-65.pdf

Serón, S., Muñoz, S. y Lanas, F. (2010). Nivel de actividad física medida a través del cuestionario internacional de actividad física en población chilena. Revista Médica de Chile, 12(8), 1232-1239. Recuperado de: http://www.scielo.cl/pdf/rmc/v138n10/art\%2004.pdf

Straub, R. (2 Ed.) (2007). Health Psychology. A Biopsychosocial Approach. Nueva York, Estados Unidos de America: Worth.

Sterud, T., Hem, E., Lau, B. \& Ekeberg, Ø. (2011). A comparison of general and ambulance specific stressors: predictors of job satisfaction and health problems in a nationwide one-year follow-up study of Norwegian ambulance personnel. Journal of Occupational Medicine and Toxicology, 6(10), 6-10. http://dx.doi.org/10.1186/1745-6673-6-10

Suk, H. \& Sukhee, H. (2011). Comparison of Stress, Social Support, and Marital Satisfaction between Married Immigrant Women in Urban and Rural Areas. Korean journal of women's health nursing, (17) 2, 99-108. http://dx.doi.org/10.4069/kjwhn.2011.17.2.99 
Torres, R. M., Lambert, M. D., \& Lawver, R. G. (2010). Predicting Secondary Agriculture Teachers' Job Stress from Selected Personal, Family, and Work-Related Characteristics. Career \& Technical Education Research, 35(2), 65-79. http://dx.doi.org/10.5328/cter35.206

Trinh, O. TH., Nguyen, N.D., Dibley, M.J., Phongsavan, P. \& Bauman, A.E. (2008). The prevalence and correlates of physical inactivity among adults in Ho Chi Minh City. BioMed Central Public Health, 8(204). http://dx.doi.org/10.1186/1471-2458-8-204

Weinberg, R., \& Gould, D. (2007). Foundations of Sport and Exercise Psychology (4 ed.). Illinois, United States: Human Kinetics.

Viana, M. S., Andrade, A. A., Back, A. R., \& Vasconcellos, D. C. (2010). Nível de atividade física, estresse e saúde em bancários. Revista Motricidade, 6(1), 19-32. Recuperado de

http://www.revistamotricidade.com/arquivo/2010_vol6_n1/v6n1a03.pdf

Victorian Health Promotion Foundation. (2010). Participation in physical activity. A determinant of mental and physical health. Research summary. Obtenido de http://www.vichealth.vic. gov.au/ /media/ResourceCentre/PublicationsandResources

Recepción: 7 de octubre del 2015

Corrección: 1 de junio del 2016

Aceptación: 15 de junio del 2016

Publicación: 30 de julio del 2016

1 Christian Alberto Azofeifa Mora. El autor es Máster en Salud Integral y Movimiento Humano por la Escuela de Ciencias del Movimiento Humano y Calidad de Vida, Universidad Nacional. Se desempeñó como profesor de Educación Física en el Ministerio de Educación Pública. Actualmente labora como académico de la Escuela Ciencias del Movimiento Humano y Calidad de Vida de la Universidad Nacional de Costa Rica.

Luis Solano Mora. Académico de la Escuela de Ciencias del Movimiento Humano y Calidad de Vida, Universidad Nacional

Jorge Salas Cabrera. Doctor en Educación por la Universidad Estatal a Distancia. Académico de la Escuela de Ciencias del Movimiento Humano y Calidad de Vida, Universidad Nacional

Héctor Fonseca Schmidt: Master en Salud Integral y Movimiento Humano por la Escuela de Ciencias del Movimiento Humano y Calidad de Vida, Universidad Nacional. Docente universitario, sicopedagogo y director administrativo del Colegio ILPPAL. Actualmente se desempeña como investigador y docente en las áreas de psicomotricidad y psicosociología de la salud en el marco del Programa Psicomotricidad Infantil (PSICOMI). 